\title{
The Influence of Sound Source Directivity on Acoustics Parameters Distribution in Kraków Opera House
}

\author{
A. Golaś, K. Suder-DębSka and R. FilipeK* \\ Department of Mechanics and Vibroacoustics, AGH — University of Science and Technology \\ al. Mickiewicza 30, 30-059 Kraków, Poland
}

\begin{abstract}
Determining of Kraków Opera House's basic acoustics parameters by using numerical simulations is presented in the paper. Parameters have been obtained by using numerical simulation methods. Sound sources have been created by means of FEA, however acoustic field distributions have been analysed by geometrical methods. Apart from the room parameters definition, it is necessary to determine sound source parameters like acoustic power level and directivity pattern. In the simplest case, the sound source can be assumed as omni-directional point source. However it does not reflect most real-live sources precisely. When the literature and databases do not contain any information about the sound source directivity pattern, it can be obtained numerically using FEA. First, the sound source model is created, and then results from its spherical boundary are used to define the source in a program based on geometrical methods. Here have been analysed several distributions of acoustic parameters like: Direct Energy, Sound Pressure Level (SPL), Clarity index (C80) etc. The results indicate that influence of sound source directivity on acoustics parameters changes is essential.
\end{abstract}

PACS numbers: 43.55.Ka, 43.20.Dk, 43.40.Rj

\section{Introduction}

During the design of rooms, which must meet high requirements as regards acoustic quality, it is important to model sound propagation from a sound source to listener. Subjective opinions can turned out unreliable, so there are defined objective parameters, which can be calculated numerically with the use of geometrical methods. Apart from the room parameters definition, it is necessary to determine sound source parameters like acoustic power level and directivity pattern [1]. In the simplest case, the sound source can be assumed as omni-directional point source. However it doesn't reflect the most of real-live sources precisely. When the literature and databases do not contain any information about the sound source directivity pattern, it can be obtained numerically as in Sect. 3 .

In the preliminary analysis phase a lot of different directional sound sources were considered. The first assumption was the sound source has impulse and mechanical character. The second assumption was the source is loud enough to obtain proper sound pressure level in the room. Both criterion are fulfilled by gongs and bells, which according to unique and specific sound are often used to signalling purposes. The bell was chosen to the further study. It was because of its greater structural stiffness, in comparison to the gong, which allow geomet-

\footnotetext{
* corresponding author; e-mail: roman.filipek@agh.edu.pl
}

ric and material nonlinearities to be neglected [2]. Its directivity pattern at the first resonance frequency is more interesting than similar pattern for the gong.

The influence of the sound source directivity on acoustics parameters distribution has been analysed using several sources with the same sound power and different directional patterns: omni-directional and extremely directional. The analysis was performed on several distributions of acoustic parameters like: Direct Energy, Sound Pressure Level (SPL), Clarity index (C80) etc. - on audience of Kraków Opera House.

\section{Kraków Opera House}

New building of Kraków Opera House was committed to use on December 2008. The total volume of the hall is about $12700 \mathrm{~m}^{3}$. Basing on the technical documentation a model of the discussed hall was designed (Fig. 1). Then specified materials were attributed to the particular surfaces (comparable to these in real conditions) with its reverberation absorption coefficients.

According to references of spectators the quality of sound in the hall is very good. Numerical simulations of acoustic field in Operas' hall were performed to verify their subjective opinion. The analysis was performed for a full hall (about 700 spectators). Background noise in the hall was assumed on the level of $30 \mathrm{~dB}$. The analysis was carried out with use of the ray tracing technique, in which 100000 rays were used per every source and up to 6 surface reflections were examined. Results of numerical 

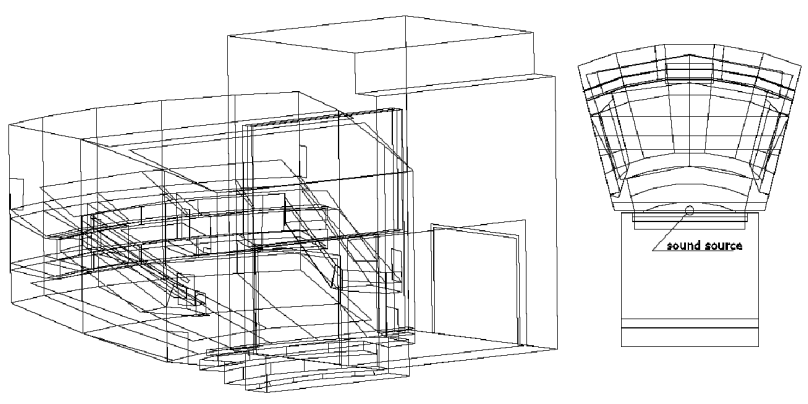

Fig. 1. Grid of the hall.

simulations deviate from results of measurements in the limit of several percent [3].

Simulations were carried out in room acoustic modelling program EASE (Enhanced Acoustic Simulator for Engineers) based on geometrical methods - in this case - ray tracing technique. Acoustic parameters RaSTI (Fig. 2) and \%Alcons have been calculated to check speech intelligibility in compartment. Results are in accordance with observations in real hall- RaSTI achieves values over 0.65 which is a very good value, \%ALcons achieves values about $5 \%$, which shows that speech intelligibility in room is very good too [4].

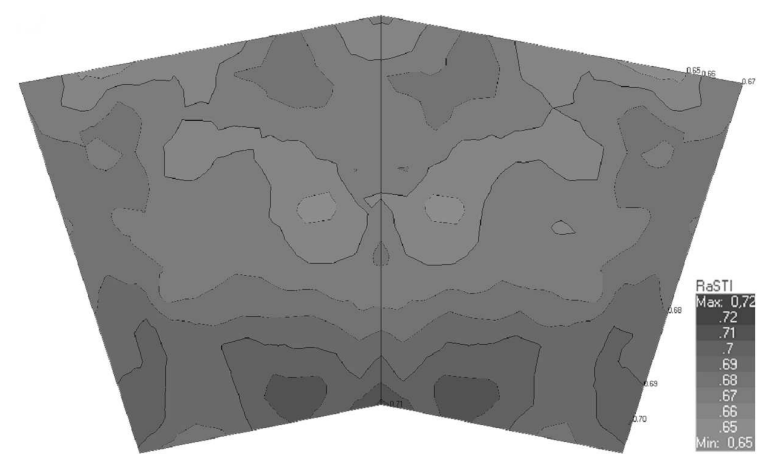

Fig. 2. RaSTI distribution on the audience in Kraków Opera House.

\section{Sound source numerical model}

The bell was assumed as the extremely directional sound source. Since the Middle Ages in the Western culture bells have had a tulip shape. More then a century ago, Lord Rayleigh assumed that the profile of a (thin) bell could be approximated by part of a hyperbola with its pole at the bell's shoulder [5, 6]. He explained why all concave bells have the first mode of vibrations named hum $(2,0)$ and why, as $m$ becomes larger mode is replaced by a second $(m, 1)$.

The simplification of the model comprises small displacements and linear behaviour, which is comparable to reality due to great stiffness of the structure.

The geometry of the bell [7] was parameterized and dimensioned with the ratios of the mouth diameter.
The material, i.e. bronze, was accepted as isotropic with the following parameters: the Young module of $10.5 \times 10^{10} \mathrm{~Pa}$, the Poisson ratio of 0.33 and density of $8600 \mathrm{~kg} \mathrm{~m}^{-3}$. The bell model was created by means of FEA and ANSYS software. The bell surface was modelled using eight node Shell 281 elements. The mesh laid evenly on the surface consisted of about 850 tetrahedral elements of the appropriate thickness in nodes located at the same height. During the first step of calculations, the bell mouth diameter was assumed from 0.1 to $1 \mathrm{~m}$ and the first modal frequency was determined. The diameter $0.253 \mathrm{~m}$ which corresponds to the frequency close to $1 \mathrm{kHz}$ was chosen. The weight of this structure (without the crown) was approximately $10 \mathrm{~kg}$. The next step of analysis was to determine sound radiation of the bell. Therefore, as shown in Fig. 3a, the acoustic volume [8] as the sphere of $0.5 \mathrm{~m}$ radius was added to the bell surface. The acoustic medium was air of density of $1.2 \mathrm{~kg} \mathrm{~m}^{-3}$ and sound velocity of $343 \mathrm{~m} \mathrm{~s}^{2}$. The volume was filled with tetrahedral and four-node Fluid30 acoustic elements. The Fluid130 elements that conserve the Sommerfeld condition causing the complete sound radiation from the system (with no reflections from the external surface) were situated at the external sphere surface. The structure and acoustic coupling was considered within a single computational step during calculations. This assumption was possible because the applied Fluid30 elements were in the contact with the bell surface and they had (besides pressure) extra degrees of freedom as displacements. The coupling of displacement field and acoustic field occurs in a matrix equation describing the element.
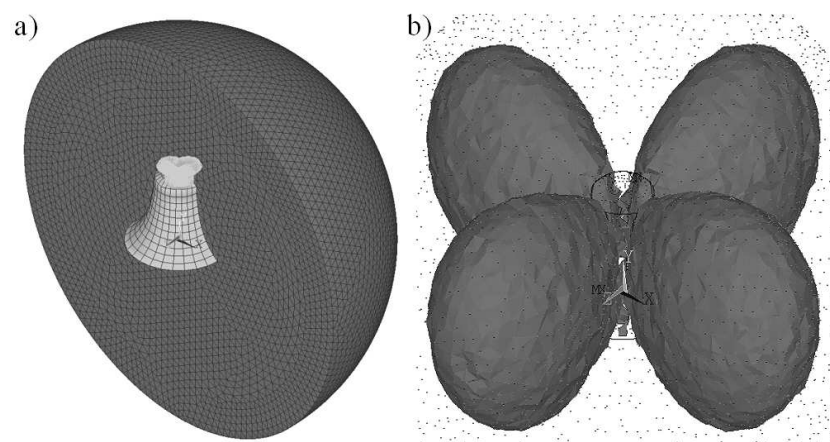

Fig. 3. (a) FE coupled field model, (b) SPL isosurface $64 \mathrm{~dB}$ for resonance frequency $1 \mathrm{kHz}$.

The key element of the analysis is always a determination of the boundary conditions. The velocity of vibrations is specified usually during a stroke. In this model the unitary harmonic force, with frequency $1 \mathrm{kHz}$, was applied to the node. The single point from the spectral transfer function was obtained.

The obtained distribution of the acoustic field for a hum frequency of the bell had features of significant irregularity. The sound radiation occurs mainly in a horizontal plane of the bell (in directions perpendicular to 
the side bell walls) and it is the most intense in four directions according to vibration antinodes. The observed directivity is similar to the quadrupole sound source.

The directivity pattern was obtained by the sound pressure level measurement in every node at the model spherical boundary. These values with adequate nodal coordinates were transferred to Matlab. The data, relative to the sound source power level, were interpolated at the points specified by Ease Loudspeaker Model [4]. This format allows to define attenuation data for $1 / 3$ octave band, measured at $5^{\circ}$ increments for a quarter, half or full sphere.

\section{Directivity pattern and acoustics parameters distribution}

To check how directivity pattern can influence the acoustics parameters distributions in the room, simulations were carried out for three types of sound sources, which had the same sound power level of $81 \mathrm{~dB}$, and were situated in the same place - in front of the stage and on its centre. Sound sources had different directivity patterns - an omni-directional source and two extremely directional sources (a bell). Second extremely directional source was produced by rotation 45 degrees of the first source. The results related to SPL are shown in Figs. 4-6. and concerning C80 are shown in Figs. 7-9.

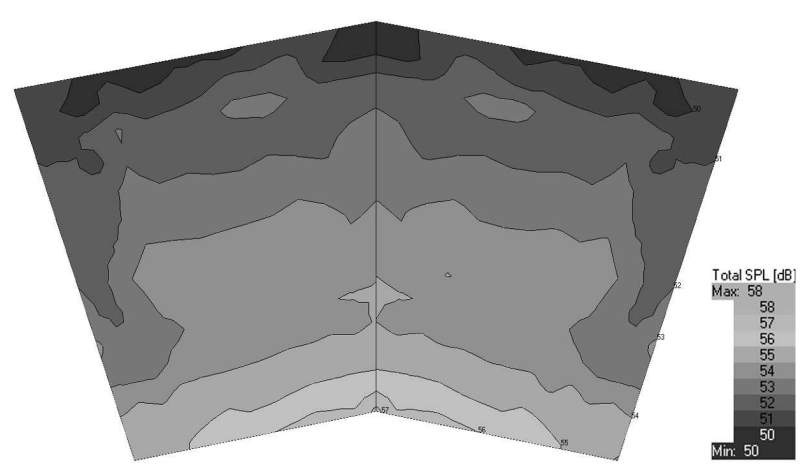

Fig. 4. SPL distribution for omni-directional source.

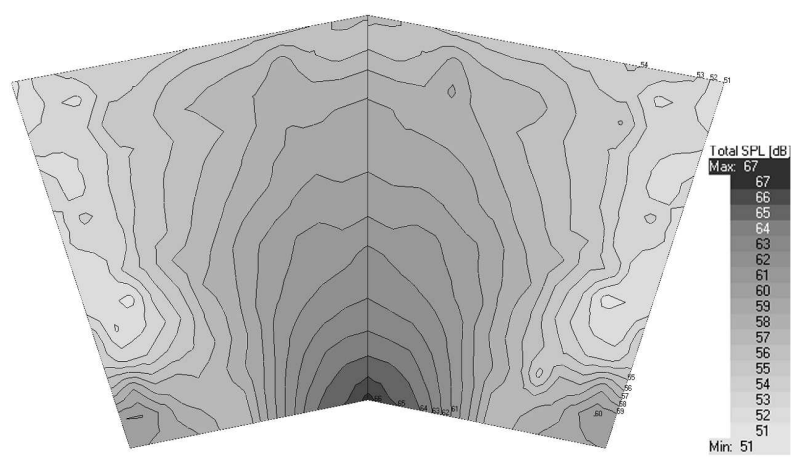

Fig. 5. SPL distribution for first extremely directional source.

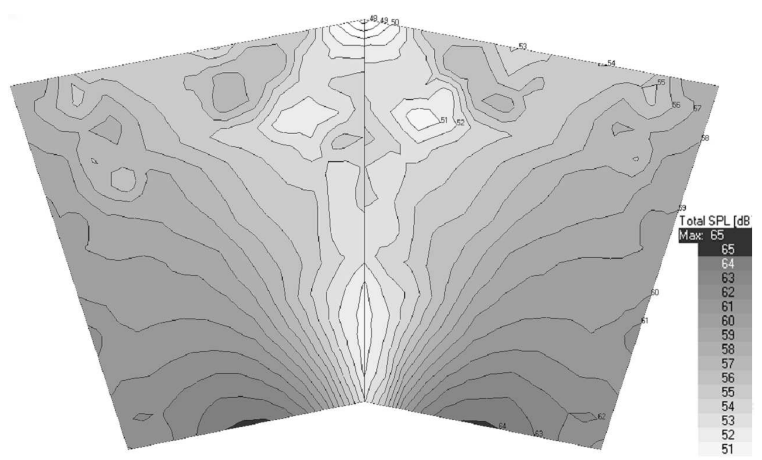

Fig. 6. SPL distribution for second extremely directional source.

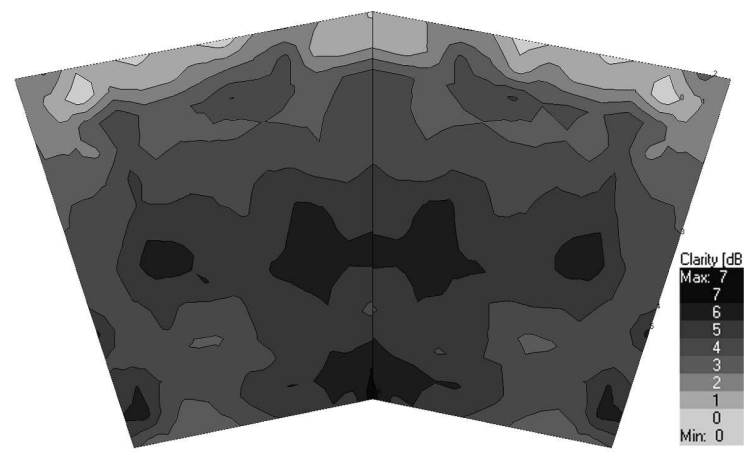

Fig. 7. C80 distribution for omni-directional source.

The omni-directional source has been found to provide very uniform Direct Energy distribution. However the bells had distributions with clear areas, where sound has been louder and areas, where sound has been clearly stiller. The source radiation directions have been visible at distributions of these areas at measurement surface. Appearance of SPL distributions has been similar, but influence of the room has been noticeable by increase values about $5 \mathrm{~dB}$ and more uniform distributions. However for omni-directional source SPL has obtained values from $50 \mathrm{~dB}$ to $58 \mathrm{~dB}$, so difference between extremely values

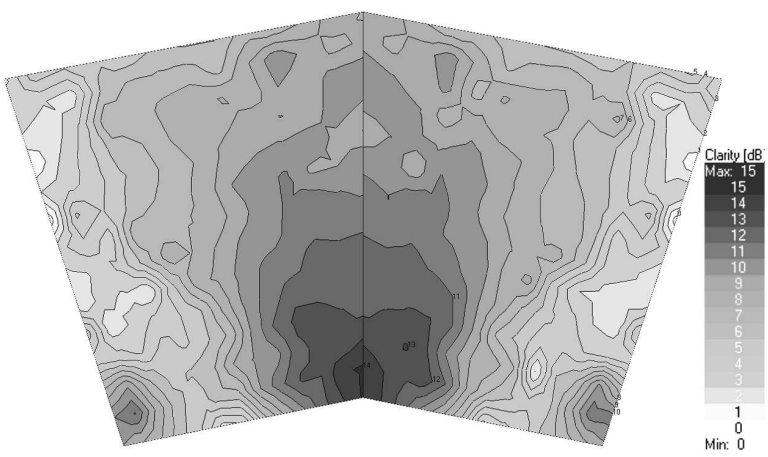

Fig. 8. C80 distribution for first extremely directional source. 


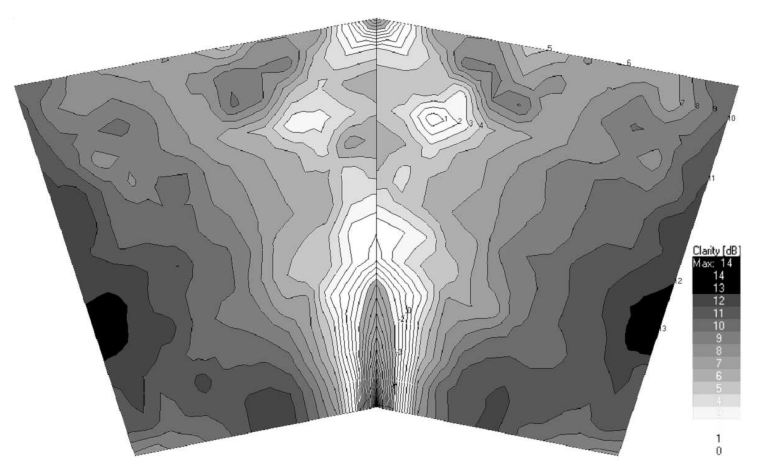

Fig. 9. C80 distribution for second extremely directional source.

has been equal to $8 \mathrm{~dB}$. Obtained extremely values for the first directional source were from $51 \mathrm{~dB}$ to $67 \mathrm{~dB}$, and for the second directional source - from $51 \mathrm{~dB}$ to $65 \mathrm{~dB}$. In the first case difference between extremely values has been equal to $16 \mathrm{~dB}$, in the second case difference has been equal to $14 \mathrm{~dB}$.

The source radiation directions has been noticeable at C80 distributions, too. Parameter histograms have been obtained in addition to computation of acoustics parameter distributions. The obtained result for omni-directional source $\mathrm{C} 80$ values, from $0 \mathrm{~dB}$ to $7 \mathrm{~dB}$, have shown that the Operas' hall is good for classic and modern music. For extremely directional sources C80 had similar values, but for a first source about $18 \%$ of area had values over $9 \mathrm{~dB}$ and for a second source about $10 \%$.

\section{Summary}

The results have shown that the acoustic field in the room can be created by the sound sources directivity pattern. Therefore the areas which have very good quality of sound and areas of silence could be created. Adequately sound sources arrangement can cause compensation of acoustics parameters distributions at interesting area. Sound source energy can be maximally utilized. However proper sound in the room can be achieved by the source rotation or changes in its localization. For sound sources, which have the same sound power but different directivity patterns, higher SPL values are obtained for extremely directional sources. Rotation of directivity pattern by 45 degree causes decrease of C 80 values (over $9 \mathrm{~dB}$ ) about $8 \%$. Obtained results can be very useful in a further experiment planning, especially for selecting a proper localization of measurement points.

The numerical calculations were carried out in the Academic Computer Centre CYFRONET AGH within the grant number MNiSW/IBM_BC_HS21/AGH/066/ 2007.

\section{References}

[1] H. Kuttruff, Room Acoustics, Spon Press, London 2000.

[2] N.H. Fletcher, Rep. Prog. Phys. 62, 723 (1999).

[3] A. Gołaś, K. Suder-Dębska, Archives of Acoustics, Vol. 34 no. 3, 273 (2009).

[4] EASE 4.1 Users Manual, Acoustic Design Ahnert, Berlin.

[5] L. Rayleigh, The London, Edinburgh and Dublin Philosophical Magazine and Journal of Science 5, (1890).

[6] R. Perrin, G.M. Swallowe, Rayleigh's Bell Model Revisited. Stockholm Music Acoustics Conference, Stockholm, Sweden 2003.

[7] A. Gołaś, R. Filipek, Archives of Acoustics, Vol. 34 no. 4 (2009).

[8] R. Filipek, J. Wiciak, The European Physical Journal - Special Topics 154, 57 (2008).

[9] L. Wang, M. Vigeant, Applied Acoustics 69, (2008).

[10] D. Davis, E. Patronis, Sound System Engineering, Elsevier Inc., Oxford 2006.

[11] Theory Reference (Release 11.0 Documentation for $A N S Y S)$. 